\title{
Zuverlässiges und unzuverlässiges Erzählen in Friedrich Dürrenmatts Kriminalromanen (1952-1958)
}

\author{
Uwe Spörl
}

Zusammenfassung Der Beitrag beschäftigt sich mit Friedrich Dürrenmatts Kriminalromanen Der Richter und sein Henker, Der Verdacht und Das Versprechen. Er vertritt die These, dass der erste und der letzte dieser Romane unzuverlässig erzählt werden, beide mimetisch und täuschend unzuverlässig, Der Richter und sein Henker heterodiegetisch bzw. erzählerlos, Das Versprechen homodiegetisch. Die These wird nach einer Bestimmung von unzuverlässigem Erzählen in Kriminalliteratur begründet und für beide Romane belegt. Sie wird darüber hinaus eingesetzt, um die Frage der Gattungszuordnung der beiden Texte als Kriminal- bzw. Antikriminalromane zu klären, und sie wird mit der gattungsübergreifenden Poetik Dürrenmatts in den fünfziger Jahren korreliert. Abschließend wird geklärt, warum der zweite der drei Romane, Der Verdacht, ohne unzuverlässiges Erzählen operiert.

Friedrich Dürrenmatts kurze Romane Der Richter und sein Henker (1952), ${ }^{1}$ Der Verdacht $(1953)^{2}$ und Das Versprechen $(1958)^{3}$ gelten einerseits als Kriminalromane, und das sicherlich nicht zu Unrecht, auch angesichts des Erfolgs beim

\footnotetext{
${ }^{1}$ Der Roman erschien zuerst zwischen Dezember 1950 und März 1951 im Schweizerischen Beobachter, 1952 dann als Buch im Benziger Verlag. Zitiert wird im Folgenden aus der dreißigbändigen Werkausgabe von 1980.

${ }^{2}$ Auch dieser Roman erschien zuerst im Schweizerischen Beobachter (zwischen September 1951 und Februar 1952), dann 1953 im Benziger Verlag. Zitiert wird im Folgenden wiederum aus der Werkausgabe.

${ }^{3}$ Dieser Roman erschien erstmals 1958 im Verlag der Arche. Zitiert wird auch hier aus der Werkausgabe.
}

\section{U. Spörl ( $₫)$}

Fachbereich 10: Sprach- und Literaturwissenschaften,

Universität Bremen,

Bremen, Deutschland

E-Mail: uwe.spoerl@uni-bremen.de

M. Aumüller und T. Kindt, Der deutschsprachige Nachkriegsroman und die Tradition des unzuverlässigen Erzählens, Abhandlungen zur Literaturwissenschaft, https://doi.org/10.1007/978-3-476-05764-8_6 
Publikum. Dieses kennt bis heute mindestens den erstgenannten Roman und den mit dem drittgenannten verknüpften Film Es geschah am hellichten Tag (1958). Sie gelten andererseits, gerade in der den Krimi geringschätzenden Literaturwissenschaft, auch als „Anti-Kriminalromane“ (Waldmann 1971), „Beinahekrimis“ (Thielking und Vogt 2014 sowie Vogt 2014), als fundamentale „Kritik am Kriminalroman“ (Tschimmel 1981) ${ }^{4}$ oder als „Subversion oder Dekonstruktion des Detektivschemas“" (Richter 1993, S. 141). Auch das hat einiges für sich, etwa wegen des Untertitels, den Dürrenmatt dem Versprechen gegeben hat - „Requiem auf den Kriminalroman“ (Dürrenmatt 1980c) -, oder wegen der natürlich feststellbaren und immer wieder festgestellten Abweichungen der drei Romane von dem, was man als Konventionenbestand des Genres Kriminalroman in den fünfziger Jahren annehmen darf.

Eine dieser Abweichungen, die Der Richter und sein Henker und Das Versprechen betrifft, ist bislang von der Forschung aber nicht als solche erkannt und beschrieben worden. Dies soll hier nun geschehen. Ich argumentiere im Folgenden für die These, dass beide Romane unzuverlässig erzählt werden, beide mimetisch unzuverlässig, beide täuschend unzuverlässig, beide mit geschlossener, also die erzählte Welt stabilisierender Funktion, ${ }^{5}$ Der Richter und sein Henker heterodiegetisch bzw. erzählerlos, Das Versprechen homodiegetisch.

Nach einer Bestimmung von unzuverlässigem Erzählen in Kriminalliteratur (1) werde ich die These für beide Romane belegen (2 und 3). Im Anschluss daran wende ich mich drei Fragen zu, die sich aus diesem Befund ergeben: Was bedeutet er für die Frage der Gattungszuordnung der beiden Texte als Kriminal- bzw. Antikriminalromane? (4) Was bedeutet er für die Poetik Dürrenmatts (in den fünfziger Jahren)? (5) Und: Wieso wird in Der Verdacht, dem dritten, zwischen den beiden anderen veröffentlichten Roman nicht unzuverlässig erzählt? (6).

\section{Unzuverlässiges Erzählen in Kriminalliteratur}

„Ein Erzähltext ist genau dann täuschend (unzuverlässig) erzählt, wenn der Text seinen Lesern (vorübergehend) gute Gründe für falsche Annahmen über fiktive Tatsachen gibt [Hervorhebung im Original]" (Köppe und Kindt 2014, S. 239). So bestimmen Tilmann Köppe und Tom Kindt das Konzept des täuschenden unzuverlässigen Erzählens in ihrer Erzähltheorie-Einführung, die ich als Ausgangspunkt wähle, da sie mir nicht nur generell zutreffend zu sein scheint, sondern für die konkreten Zwecke meiner Untersuchung auch passend und anwendbar.

\footnotetext{
${ }^{4}$ Tschimmel (1981, S. 186) macht insbesondere Das Versprechen als den Roman Dürrenmatts aus, der „dem ,klassischen“ Kriminalroman den tödlichen Dolchstoß“ versetzt.

${ }^{5}$ Zur Unterscheidung von geschlossener, die erzählte Welt stabilisierender und offener, destabilisierender (Interpretations-)Funktion unzuverlässig erzählter Texte vgl. insbes. Aumüller (2018, S. 133).
} 
Ergänzen möchte ich diese Bestimmung um den folgenden Hinweis: Mit Kindt (2008, S. 65-67), Simone Lang (2016, S. 39), Theresa Heyd (2011) und anderen nehme ich an, dass zuverlässiges und unzuverlässiges Erzählen im Rahmen der pragmatischen Kommunikationsprinzipien von H. Paul Grice (1979) zu bestimmen ist. Demnach will die Erzählung als Werk verstanden werden und folgt dem Kooperationsprinzip der Kommunikation, sie weicht aber, wenn sie unzuverlässig erzählt, als fiktionale Erzählung von diesem Prinzip in quantitativer, qualitativer, relationaler und/oder modaler Hinsicht ab.

Für den konkreten Fall von Kriminalromanen müsste man also wohl ein gattungsspezifisches kommunikatives Kooperationsprinzip annehmen. Denn Kriminalromane sind ja als Spannungs- und Unterhaltungsliteratur gerade darauf angewiesen, dass die Erzählung ihrer Leserschaft bestimmte Informationen zum Fall und seiner fortschreitenden Aufklärung in angemessener Weise und passendem Umfang zu einem bestimmten Zeitpunkt gibt oder vorenthält. Kurz, ich plädiere - mindestens für den Kriminalroman - für eine gattungssensitive Bestimmung von (un)zuverlässigem Erzählen. ${ }^{6}$

Was erwartet also die Leserschaft von einem in den fünfziger Jahren in der Schweiz veröffentlichten Kriminalroman? Sie erwartet sicherlich einen Detektivroman, in dem, so schon Richard Alewyn in seiner Anatomie des Detektivromans, der Erzähler ,am Anfang [...] schon alles“ weiß, aber nicht erzählt, ,der Leser [indes] noch nichts“" weiß (Alewyn 1998, S. 54), sodass sich im Lauf der Erzählung „der Abstand zwischen Erzähler und Leser“ verringert, bis am Ende der Leser „nun ebenfalls alles [weiß]“ (Alewyn 1998, S. 55). ${ }^{7}$

Anders gesagt: Das Zurückhalten von Informationen durch den Erzähler gehört zum ,Spiel ${ }^{\circ}$ des Krimis ${ }^{8}$ und der ihm eigenen Erzeugung von (kognitiver) Spannung. ${ }^{9}$ Dabei folgt der Leser in seinem Wissenserwerb, der in der vollständigen Lösung des Falls mündet, dem Ermittler, der wie er angesichts des zu lösenden Rätsels,

\footnotetext{
${ }^{6}$ Eine ähnliche Überlegung findet sich in Ligensa (2008, S. 60): „Konventionen des Kriminalgenres“ oder andere „Schemata“ können, so Annemone Ligensa, erzählerische Zuverlässigkeit suggerieren. Vergleichbar argumentiert auch Sonja Klimek, wenn sie die „Affinität zwischen Phantastik und Unzuverlässigkeit“ (Klimek 2018, S. 31) der in aller Regel die erzählte Welt nicht destabilisierenden Erzählweise des Kriminalromans (Klimek 2018, S. 35) gegenüberstellt, bevor sie am Beispiel der Krimis von Tana French zeigt, wie sich Hybridbildungen zwischen kriminalliterarischem und phantastischem Genre bzgl. der Zuverlässigkeit des Erzählens verhalten, und zudem plausibel machen kann, dass die für beide Genres typische Serialität Auswirkungen auf die (Un-)Zuverlässigkeit des Erzählens haben kann (Klimek 2018, S. 45, 47).

${ }^{7}$ Ähnlich deutlich auch der genrebestimmende, zudem narratologisch fundierte Befund Uwe Lindemanns: „Der Informationsstand von Detektiv und Leser ist vor der abschließenden Lösung also derselbe“" (Lindemann 2002, S. 45).

${ }^{8}$ Zum Spiel der Kriminalliteratur als Genre vgl. insbesondere Suits (1998), zur Bedeutung des Spiels für Dürrenmatts Poetik (auch in Bezug auf seine Kriminalromane) vgl. Gasser (2014).

${ }^{9}$ Zum Konzept der Spannung „für rein kognitive Aktivierungen [...], also zum Beispiel in Bezug auf das kontinuierlich rätsel- und problemlösende Verstehen einer literarischen Handlung, das durch bestimmte Informationsvergabestrategien im literarischen Text gezielt manipuliert werden kann“, vgl. insbesondere Mellmann (2016, S. 166).
} 
welches das aufgefundene Mordopfer darstellt, ebenfalls noch nichts, durch seine Aufklärungstätigkeit aber am Ende alles den Fall Betreffende weiß. Die Erzählung folgt also in ihrer Mitteilung von fallbezogenem Wissen dem Ermittler und dem Ermittlungsprozess: „Leser und Detektiv müssen gleichwertige Möglichkeiten haben, das Geheimnis zu lösen“ (Van Dine 1971, S. 143), lautet entsprechend die erste von Zwanzig Regeln für das Schreiben von Detektivgeschichten Van Dines aus dem ,Golden Age‘ des Genres.

Ein Detektivroman ist also dann täuschend unzuverlässig erzählt, wenn er vor dem Hintergrund dieser Genre-Standards der Informationsvergabe seinen Lesern vorübergehend gute Gründe für falsche Annahmen über fiktive Tatsachen gibt. Die (sich im Laufe der Erzählung vermindernde) Beschränkung des Erzählers auf den (je aktuellen, sich im Laufe der erzählten Ermittlungen erhöhenden) Wissensstand des Ermittlers rechtfertigt also nicht die Annahme unzuverlässigen Erzählens im konventionellen Detektivroman. Wenn jedoch von dieser Konvention so abgewichen wird, dass die Leser zu falschen Annahmen über fiktive Tatsachen etwa über den Ermittler und seine Ermittlungsfortschritte - veranlasst werden, liegt unzuverlässiges Erzählen im Detektivroman vor.

\section{Unzuverlässiges Erzählen in Der Richter und sein Henker}

Damit komme ich zu Dürrenmatts erstem Kriminalroman Der Richter und sein Henker. Der heterodiegetisch erzählte Roman setzt ganz erwartungsgemäß mit dem Fund einer Leiche ein: An einer Landstraße unweit des Bielersees wird ein Toter aufgefunden. Ulrich Schmied, ein Berner Polizist, ist erschossen worden (Dürrenmatt 1980a, S. 11 f.). Von wem und warum soll sein Vorgesetzter, „Kommissär Bärlach“ (Dürrenmatt 1980a, S. 13) aufklären, gemeinsam mit Tschanz, der ihm als Assistent zugewiesen wird.

Der Leser hat hier also aufgrund der Genrekonventionen des Detektivromans gute Gründe anzunehmen, dass Bärlach und Tschanz, die beiden Ermittler, vor Beginn ihrer Ermittlungen nicht wissen, wer Schmied getötet hat.

Gegenüber seinem Vorgesetzten Dr. Lutz erklärt Bärlach zwar schon früh, jemanden im Verdacht zu haben (Dürrenmatt 1980a, S. 18). Um wen es sich handelt, will Bärlach aber weder seinem Chef noch seinem Mitarbeiter sagen, sodass es natürlich auch der Leser nicht erfährt. ${ }^{10}$ Überhaupt verhält sich Bärlach

\footnotetext{
${ }^{10}$ Bärlach und Dr. Lutz debattieren ständig über kriminalistische Methoden. Lutz favorisiert die modernen, in den USA bereits realisierten Standards moderner Ermittlungsarbeit, Bärlach kommt gut auch ohne derlei zurecht. Diese Debatten und die kriminalistischen Diskurse, auf die sie Bezug nehmen, rekonstruiert Maximilian Bergengruen. In seiner Ablehnung wissenschaftlicher Methoden der Kriminalistik erweist sich, so Bergengruen (2014, S. 60), der „kriminalistisch rückwärtsgewandte [...] Bärlach“ als der „letzte Bewahrer des klassischen Kriminalromans“.
} 
etwas seltsam, aber auch das dürfte den Erwartungen der Leser an einen RomanDetektiv durchaus entsprechen: Er hat aus Schmieds Wohnung eine Mappe entwendet, leugnet das aber gegenüber Tschanz (Dürrenmatt 1980a, S. 27). Bärlach wird im Laufe der Ermittlungen von einem Hund angegriffen, ,ein entfesseltes Ungeheuer an Kraft und Mordlust“ (Dürrenmatt 1980a, S. 37), den Tschanz erschießt, um seinen scheinbar unbewaffneten Vorgesetzten zu retten. Ein paar Seiten später erfährt der Leser aber, dass der nun allein nach Hause zurückgekehrte Bärlach seine Waffe aus der Manteltasche holt und die ,dicken Tücher[]“ abnimmt, die er um seinen linken Arm gewickelt hat, ,wie es bei jenen Brauch ist, die ihre Hunde zum Anpacken einüben“ (Dürrenmatt 1980a, S. 48).

Diese Indizien für den miträtselnden Leser auf einen Ermittler, der mehr weiß, als er seinen Kollegen gegenüber preisgibt, gehen aber - genretypisch unter in einer Vielzahl weiterer Informationen zum Fall. Denn die Aufklärungsgeschichte $^{11}$ und mit ihr die Ermittlungen nehmen rasch Fahrt auf und werden bald heikel, weil politisch: Schmied, der Ermordete, hat offenbar unter Pseudonym an den Abendgesellschaften eines gewissen Gastmann teilgenommen, zusammen mit Künstlern - darunter auch ein Schriftsteller -, vor allem aber mit Schweizer Industriellen und „,Angehörige[n] einer fremden Gesandtschaft"“ (Dürrenmatt 1980a, S. 54), wie von Schwendi, Gastmanns Anwalt, erklärt. Der Verdacht liegt also nahe, dass Gastmann Schmied als Polizisten enttarnt hat und hat umbringen lassen. Gastmann ist ohnehin alles zuzutrauen. Er sucht Bärlach privat auf, und im von der Erzählung wiedergegebenen Gespräch (Dürrenmatt 1980a, S. 66-73) zwischen den beiden stellt sich Folgendes heraus: Bärlach kennt Gastmann schon seit 40 Jahren. Dieser ist nicht nur ein politisch-geschäftlicher Strippenzieher, sondern auch ein Berufsverbrecher, den Bärlach seit ihrer ersten Begegnung jagt und belangen will. Damals hat Gastmann ohne Skrupel vor Bärlachs Augen einen Mord begangen, den dieser aber nicht hat nachweisen können.

Gastmann kann auch der Mord an Schmied nicht nachgewiesen werden, schon weil er ihn, wie sich herausstellt, trotz aller Verdachtsmomente nicht begangen hat. Bärlach erklärt ihm aber, ihn, den mit polizeilichen und juristischen Mitteln nicht zu fassenden ,master criminal“, zu ,,,richten““ und ,,,zum Tode verurteilt“" zu haben (Dürrenmatt 1980a, S. 100).

Und so geschieht es dann auch: Tschanz, der sich profilieren will, wird zum „Henker" und erschießt Gastmann mutwillig, aber offiziell in Notwehr beim Versuch, diesen zu verhaften (Dürrenmatt 1980a, S. 104). Für Dr. Lutz ist der Fall damit abgeschlossen (Dürrenmatt 1980a, S. 106). Doch Bärlach und Tschanz, die sich zum Abschluss des Romans noch einmal treffen, wissen es besser - und erklären in ihrem Gespräch sich und den Lesern, wie die tatsächliche Lösung des Kriminalfalls mit allen Indizien, die sich während der Ermittlungen ergeben haben, zusammenstimmt:

\footnotetext{
${ }^{11}$ Zur für den Detektivroman wesentlichen Unterscheidung von Aufklärungs- und Verbrechensgeschichte vgl. Lindemann (2002).
} 
Tschanz hat Schmied als Konkurrenten in der Polizeihierarchie getötet, aus Neid und Profilierungssucht. Und er weiß bzw. hat von Anfang an geahnt, dass Bärlach das weiß (Dürrenmatt 1980a, S. 111). Und Tschanz erkennt nun auch, wie der Leser, dass Bärlach dieses Wissen genutzt hat, um Tschanz auch zum Mörder am verhassten Gastmann werden zu lassen:

Tschanz taumelte. Es überlief ihn eiskalt. „Da haben Sie mich und Gastmann aufeinander gehetzt wie Tiere!“ „Bestie gegen Bestie“, kam es unerbittlich vom andern Lehnstuhl [Bärlachs] her. „Dann waren Sie der Richter, und ich der Henker“, keuchte der andere. „Es ist so“, antwortete der Alte. (Dürrenmatt 1980a, S. 115)

Dass dieses Vorgehen Bärlachs, der krankheitsbedingt ohnehin aus dem Polizeidienst ausscheiden und bald sterben wird, moralische Fragen aufwirft, ist klar und ist immer wieder in der Forschung diskutiert worden. Klar ist aber auch, dass dieses Ende des Romans, das einerseits alle im Laufe der Erzählung, also der Aufklärungsgeschichte erwähnten Spuren und Indizien zu einem schlüssigen Gesamtbild der Verbrechensgeschichte zusammensetzt, andererseits für den Leser überraschend ist, vollständig den Konventionen des Detektivromans entspricht.

Und klar ist auch, dass die Überraschung für den Leser zustande kommt, weil täuschend unzuverlässig erzählt worden ist. Denn beide, Bärlach wie Tschanz, wussten schon zu Beginn der Ermittlungen, wer der Mörder Schmieds ist, obwohl der Leser aus dem Konventionenbestand des Detektivromans gute Gründe für die Annahme hat, dass sie es nicht wissen.

\section{Unzuverlässiges Erzählen in Das Versprechen}

Im Roman Das Versprechen erzählt auf der ersten Ebene einer Rahmenerzählung ein namenloser Ich-Erzähler von einem in Chur gehaltenen Vortrag ,über die Kunst, Kriminalromane zu schreiben“ (Dürrenmatt 1980c, S. 11), und seiner nächtlichen Begegnung mit „Dr. H., de[m] ehemalige[n] Kommandanten der Kantonspolizei Zürich“ (Dürrenmatt 1980c, S. 12) an der Hotelbar. Dieser bietet dem Ich-Erzähler an, ihn am nächsten Morgen mit dem Wagen zurück nach Zürich zu nehmen. Auf dieser Fahrt hält Dr. H. an einer heruntergekommenen Tankstelle an, vor der ein offenbar verwirrter Alter sitzt und brummelt: „,Ich warte, ich warte, er wird kommen“" (Dürrenmatt 1980c, S. 16).

Das Rätsel dieser merkwürdigen Begegnung an der Tankstelle löst Dr. H. auf der weiteren Heimfahrt im Gespräch mit dem Krimiautor und Ich-Erzähler rasch auf, ${ }^{12}$ indem er diesem erstens erklärt, dass ihn die krimispezifische „,Fiktion [...] wütend“" macht, weil ,,,[d]er Wirklichkeit [...] mit Logik nur zum Teil beizukommen““ sei und weil in Kriminalromanen ,,,der Zufall keine Rolle““ spiele,

\footnotetext{
${ }^{12}$ Dass die Tankstelle und die Begegnung dort den Status eines aufzulösenden Rätsels haben und die dieses Rätsel lösende Rahmenerzählung deshalb analytisch organisiert ist, stellt auch Wigbers (2006, S. 126 f.) fest.
} 
in der Wirklichkeit hingegen sehr wohl (Dürrenmatt 1980c, S. 18). Zweitens will und wird er ihm, um diese seine Einschätzung exemplarisch zu belegen, eine Geschichte erzählen, die Geschichte des ,Kommissärs“ Matthäi, der ,,,vor nun bald neun Jahren“" der beste Kriminalkommissar Zürichs war, ja ,,ein Genie““ (Dürrenmatt 1980c, S. 19) sogar, nun aber, wie eben gesehen, ein versoffener und völlig heruntergekommener Tankstellenbetreiber ist. Nach dieser poetologischen, metafiktionalen und metanarrativen Einrahmung, ${ }^{13}$ die im weiteren Verlauf des Romans hin und wieder aufgegriffen und dem Leser so ins Bewusstsein gerückt wird, erzählt Dr. H. seine Geschichte vom letzten Fall Matthäis, stets in doppelten Anführungszeichen, die seine intradiegetische Erzählung als wörtliche Rede markieren.

Dr. H., der Erzähler, ist dabei in seiner Binnenerzählung stets darum bemüht, seinem Gesprächspartner deutlich zu machen, woher er sein Wissen von den Ermittlungen Matthäis hat: Teilweise war er als Polizeichef selbst beteiligt und vor Ort, anderes wurde ihm zugetragen und „,rapportiert““ (Dürrenmatt 1980c, S. 46). Auch die ,Erzählgeschichte' ${ }^{14}$ also die Geschichte des Erzählens dieser Geschichte, wird klar: Die Autofahrt ist längst zu Ende, als die Binnenerzählung Dr. H.s gerade begonnen hat. Sie wird deshalb bei einem ausgiebigen Mittagessen bis weit in den Nachmittag hinein fortgesetzt.

Im Zusammenhang mit dieser Erzählgeschichte räumt der Ich-Erzähler der ersten Ebene auch bald ein, dass er „die Erzählung des redegewaltigen Alten natürlich nicht immer so wiedergegeben [hat], wie sie [ihm] berichtet wurde“", sondern dass er ,,jene Teile seiner Geschichte, die er nicht von seinem Standpunkte aus, von seinem Erlebnis her" erzählte, ,neu geformt" hat (Dürrenmatt 1980c, S. 140 f.). Anders gesagt: Die Wiedergabe des Erzählberichts des intradiegetischen Erzählers Dr. H. durch den extradiegetischen Erzähler des Rahmens ist trotz der Verlässlichkeit und authentische Wiedergabe anzeigenden Anführungszeichen unzuverlässig bzgl. der Modalität des Erzählens, da sie entgegen den intrafiktionalen Tatsachen dem Erzähler Dr. H. immer wieder Innensicht in den Ermittler Matthäi und interne Fokalisierung auf ihn zuschreibt.

So tritt etwa, um dieses Verfahren exemplarisch zu belegen, Matthäi den Eltern des Mordopfers allein gegenüber, um ihnen vom Mord an ihrer Tochter zu berichten. Dennoch verfügt Dr. H. in seiner vom Erzähler des Rahmens modifizierten Erzählung über Innensicht in Matthäi: „Matthäi wartete. Es entging ihm nichts, und er wußte auf einmal, daß er diese Szene nie mehr vergessen würde“ (Dürrenmatt 1980c, S. 32). Und Dr. H. nimmt als Erzähler auch die Perspektive des Kommissärs ein, etwa nach dem Versprechen Bärlachs den Eltern gegenüber, den Mörder zu finden: „Der Kommissär stutzte. ,Bei meiner Seligkeit‘, sagte er endlich. Was wollte er anderes“(Dürrenmatt 1980c, S. 32).

\footnotetext{
${ }^{13}$ Vogt (2014, S. 62) plädiert dafür, die Positionen Dr. H.s in dieser poetologischen Einrahmung „als Friedrich Dürrenmatts Theorie des Kriminalromans“ anzusehen. Die folgenden Ausführungen sollten zeigen, dass das nicht plausibel ist.

${ }^{14}$ Zum Konzept der Erzählgeschichte vgl. insbesondere Schmid (2005, S. 268-270).
} 
Dr. H.s Erzählung folgt damit aber natürlich den Konventionen kriminalliterarischen Erzählens mit ihrer dominanten Bezugnahme auf die Wissensbestände des Ermittlers Matthäi. Denn dieser ist der Binnenerzählung Dr. H.s zufolge der Ermittler dieser Kriminalerzählung, auch wenn er mit seinen Ermittlungen scheitert.

Der Fall, an dem Matthäi scheitert, ist dieser: Die kleine Gritli Moser ist im Wald getötet worden. Ihre Leiche findet (zufällig) der Hausierer von Gunten, den sogleich die Polizei und die Dorfgemeinschaft für den Mörder halten. Nur Matthäi ist von seiner Schuld nicht überzeugt. Zudem lässt sich Matthäi gegenüber Gritlis Eltern zum titelgebenden Versprechen hinreißen, den Täter zu finden. Von Gunten tötet sich selbst in der Haft, was allseits als Schuldeingeständnis bewertet wird. Nur Matthäi wertet die Indizien anders, fühlt sich an sein Versprechen gebunden und zudem in der Pflicht, künftige Opfer des mutmaßlich noch unbekannten Mörders zu schützen, indem er diesen aufspürt - nun als privater Ermittler in eigener Sache.

Der von ihm als Berater hinzugezogene Psychiater Locher liefert die Hypothese für die Erklärung des Mordes an Gritli und weiterer ähnlicher Morde: „,Es handelt sich nicht um einen Lustmord [...], sondern um einen Racheakt"“ (Dürrenmatt 1980c, S. 98), bei dem das kindliche Opfer eine dominante erwachsene Frau vertritt. Matthäi beschließt daraufhin, diesem Rachemörder an geeigneter Stelle einer Landstraße zwischen Graubünden und Zürich - eine Falle zu stellen. ${ }^{15}$ Er pachtet besagte Tankstelle, holt sich eine Haushälterin ins Haus, deren kleine Tochter Annemarie der ermordeten Gritli ähnelt, und wartet. Und tatsächlich scheint sein riskanter Lockvogelplan zu funktionieren: Annemarie hat wie Gritli von einem ,Zauberer" Trüffelschokoladenkugeln erhalten und sich wieder mit ihm verabredet (Dürrenmatt 1980c, S. 125 f.), sodass Matthäi sich am Ziel glaubt, die Falle nur noch zuschnappen muss.

Im Spielfilm Es geschah am hellichten Tag, dessen Drehbuch Dürrenmatt gemeinsam mit dem Regisseur Ladislao Vajda verfasst hat, ${ }^{16}$ schnappt sie bekanntlich zu, und Heinz Rühmann alias Matthäi fasst Gerd Fröbe als Kindermörder. Im Film endet die Kriminalerzählung also gattungskonform mit der Überführung des Täters. ${ }^{17}$ Im Roman wartet der Kommissär allerdings vergeblich - mindestens bis zum Tag, an dem ihn Dr. H. und der Rahmenerzähler in seiner Tankstelle aufsuchen.

\footnotetext{
${ }^{15}$ Näheres zur Geo- und Topographie dieses Ortes als Falle erläutert Wigbers (2006, S. 122 f.).

${ }^{16}$ Zum Verhältnis von Film und Roman vgl. Dürrenmatts eigenes Nachwort (Dürrenmatt 1980d) sowie Broich (1998, S. 105): „Dem Roman liegt [...] eine andere Weltsicht zugrunde als dem Film. Im Film herrschte noch die Grundprämisse des klassischen Detektivromans von der [...] Kongruenz von Denken und Sein. Im Roman dagegen scheitert Matthäi an einer Wirklichkeit, die $[\ldots]$ vom Zufall $[\ldots]$ beherrscht wird.“

${ }^{17}$ Die US-amerikanische Neuverfilmung des Romans The Pledge (2001) orientiert sich bzgl. des Endes der Geschichte am Roman - auch hier wartet der Ermittler vergeblich, dass seine Lockvogelfalle zuschnappt -, anders als im Roman wird die Aufklärung des Falls aber nur angedeutet, nicht erzählt.
} 
Der Roman endet aber gerade nicht mit diesem Scheitern des Ermittlers Matthäi, das der Erzähler Dr. H. auf das von Krimiautoren zu wenig berücksichtigte Wirken des Zufalls zurückführt. Denn auch ihm, Dr. H., hilft der Zufall, und die Aufklärungsgeschichte wird fortgesetzt und abgeschlossen: Kurz vor seiner Pensionierung wird er, wie er weiter erzählt, ans Sterbebett einer alten Dame gerufen. Diese hat in zweiter Ehe ,,, den nun auch seligen Schrott““ (Dürrenmatt 1980c, S. 152) geheiratet, ihren deutlich jüngeren Chauffeur und Gärtner. Dieser Schrott, ein sehr schlichtes Gemüt, hat zwar die Ehe mit ihr nie vollzogen (Dürrenmatt 1980c, S. 153) und sich seiner in jeder Hinsicht überlegenen und dominanten Ehefrau, die er „,Mutti““ nennt (Dürrenmatt 1980c, S. 153), vollkommen unterworfen. Für diese andauernde Erniedrigung hat er sich aber offenkundig - wie Frau Schrott in einer Mischung aus Naivität und Skrupellosigkeit angesichts ihres bevorstehenden Todes dem Dr. H. erzählt - ein Ventil gesucht und auf seinen Touren durchs Land, die sie ihm gestattet hat, kleine Mädchen getötet, eben auch Gritli. Als er aufbrechen will, um Annemarie, Matthäis Lockvogel, zu töten, kommt es zum Streit zwischen den ungleichen Eheleuten. Frau Schrott erinnert sich:

\footnotetext{
„,Da wurde ich energisch, ,Es gibt nichts, Albertchen', habe ich gesagt, ,du hast es mir versprochen, reinige auf der Stelle den Hühnerstall und gib den Hühnern ordentlich zu fressen. ' Da ist Albertchen zornig geworden, das erstemal in unserer Ehe, die doch sonst so harmonisch war, hat geschrien, ,Ich bin nur dein Hausknecht', so krank war er, und ist hinausgerannt mit den Trüffeln und dem Rasiermesser zum Buick, und schon eine Viertelstunde später hat man mir telephoniert, er sei mit einem Lastwagen zusammengestoßen und gestorben [...] “" (Dürrenmatt 1980c, S. 160)
}

Dieser ,Zufall` hat verhindert, dass Matthäis Hypothese vom Täter aus männlicher Demütigung bestätigt wird, seine Falle zum Erfolg und zur Festnahme des Täters führt. Der gescheiterte Matthäi hat für diese nachträgliche Lösung seines Falls aber längst kein Verständnis mehr (Dürrenmatt 1980c, S. 162).

Ein anderer ,Zufall ' - dass Dr. H. ans Sterbebett der Schrott gerufen wird und sie ihm beichtet - führt aber dazu, dass nicht nur der Fall Gritli Moser endgültig aufgeklärt wird, sondern erklärt auch, warum Matthäi mit seiner Falle an der Tankstelle keinen Erfolg gehabt hat. Und das geschieht ganz konform mit den Regeln des Detektivromans auf den letzten Seiten. Hier erweist sich also auch, dass die bis dahin gerechtfertigte Annahme des Lesers, Matthäi sei der Ermittler des erzählten Falls, falsch ist, zumindest ergänzungsbedürftig. Denn der Ermittler Dr. H. ist es - gemeinsam mit ,Kommissar Zufall ‘ -, der den Fall erfolgreich zum Abschluss bringt.

\section{Zur Gattung der beiden Romane}

Beide untersuchten Romane weichen also mindestens dadurch von den Konventionsbeständen des Detektivromans ab, dass sie bezogen auf die (Identität der) Ermittlerfigur unzuverlässig erzählen. In Der Richter und sein Henker wird dem 
Leser nahegelegt, der Ermittler Bärlach wisse zu Beginn der Ermittlungen so wenig wie er selbst, was falsch ist. In Das Versprechen hat der Leser während der Lektüre gute Gründe zu glauben, Matthäi sei der Ermittler und scheitere, tatsächlich löst Dr. H. als Ermittler den Fall aber auf. Sind beide Texte gleichwohl als Kriminalromane zu verstehen?

Ich plädiere für beide Romane dafür, die Frage eindeutig zu bejahen. Bei dieser Antwort ist allerdings zu berücksichtigen, dass nicht völlig klar ist, welche Gattungsbestimmungen und damit einhergehende Leseerwartungen man dem deutschsprachigen Lesepublikum und seinem Autor in den 50er Jahren zuschreiben darf. ${ }^{18}$ Umstritten in der Forschung ist etwa die Frage, welche Krimis Dürrenmatt schätzte bzw. als Vorbilder akzeptierte und ggf. adaptierte. ${ }^{19}$

Klar ist freilich, dass das scheinbar so regelfixierte Genre der Kriminalliteratur gerade im Verlauf des 20. Jahrhunderts zahlreichen Veränderungen unterlag. Die Geschichte des Kriminalromans ist also wohl beschreibbar als eine Geschichte von Abweichungen von vorausgehenden Normen, sodass das Genre immer wieder neue Spielarten und Subgenres ausgebildet hat. ${ }^{20}$ Für die erste Hälfte des 20. Jahrhunderts sind dabei wohl zwei Entwicklungen maßgeblich: erstens, vor allem in den USA, die Abkehr vom Detektivroman klassischen Zuschnitts, in dem - in den Worten Dr. H.s - die überlegene ,Logik' eines Sherlock Holmes oder Hercule Poirot dominiert, zugunsten einer ,realistischen“ Darstellung der ,Wirklichkeit'; und zweitens - in Europa, etwa bei Simenon oder Glauser - die Nutzung des Kriminalromans zur Darstellung und Durchleuchtung sozialer und kultureller Milieus.

Dass Kriminalromane also von ihnen zugrundeliegenden Regeln abweichen können, ohne deshalb zwingend außerhalb der Gattung zu stehen, kann, denke ich, vorausgesetzt werden. Dürrenmatts Das Versprechen ist - gerade im Vergleich mit dem völlig gattungskonventionellen Film Es geschah am hellichten Tag - dafür geradezu exemplarisch. Die metanarrative Rahmenerzählung ist zwar sicherlich ungewöhnlich, thematisiert aber gleichwohl nur, was im Genre ohnehin schon bekannt ist. Und die abschließende Erzählung der Witwe Schrott schließt den Fall nach allen Regeln der Kunst zu einem für den Leser überraschenden Krimiende ab. Dürrenmatts Roman mag also ein „Requiem auf den Kriminalroman“ heißen, ein Kriminalroman ist er gleichwohl.

\footnotetext{
${ }^{18}$ Vgl. dazu die diesbezüglichen Ausführungen in Vogt (2014, S. 69-72).

${ }^{19}$ Vgl. dazu Arnold (1981), der neben Wallace und Christie vor allem Simenon mit seinen Maigret-Romanen als Muster ausweist, und Riedlinger (2007, S. 127-139), der vor allem die Parallelen zwischen Glausers und Dürrenmatts Kriminalromanen betont. Ladenthin (2016, S. 388) nennt zusätzlich unter Bezugnahme auf Dürrenmatts Jugend(erinnerungen) „die in den 30er bis 50er Jahren populäre Heftreihe John Kling's Abenteuer".

${ }^{20}$ Volker Neuhaus nimmt bei seinen Ausführungen zur Gattungsgeschichte des Detektivromans, auf die ich an dieser Stelle verweisen möchte, vor allem die spielerischen Qualitäten und Dimensionen komischer und parodistischer Intertextualität in den Blick. Die Entwicklungen des Genres sind somit, so Neuhaus (2010, S. 39), nach den aus der vormodernen Gattungspoetik bekannten Prinzipien ,imitatio, variatio und aemulatio“ zu begreifen.
} 
Ein anderes berühmtes Beispiel aus der Geschichte des Kriminalromans unterstützt diesen Befund: Agatha Christie wurde 1926 gerade durch ihren Roman The Murder of Roger Ackroyd (dt: Alibi, Christie 1961) berühmt. In diesem ist es bekanntlich der Ich-Erzähler selbst, den der an Doyle geschulte Leser für eine Watson-Figur hält, der sich aber als der gesuchte Mörder entpuppt - und als täuschend unzuverlässiger Erzähler. Hier steht die Unzuverlässigkeit des Erzählens also klar im Dienst der überraschenden Auflösung des Kriminalfalls. ${ }^{21}$

Genau das trifft auch auf die beiden untersuchten Romane Dürrenmatts zu: In Bezug auf Das Versprechen habe ich das eben schon deutlich gemacht. Für Der Richter und sein Henker gilt Entsprechendes: Erst am Ende der Erzählung, in diesem Fall freilich vorbereitet von zahlreichen Indizien, erfährt der Leser nicht nur, dass der Ermittler Bärlach von Anfang an wusste, wer Schmied getötet hat, sondern er erfährt auch, wer Schmied getötet hat, nämlich Tschanz. Die scheinbare Tätersuche Bärlachs war tatsächlich also nichts anderes als die Umsetzung seines Plans, ohne direkte juristische Folgen Tschanz zur Tötung Gastmanns zu bewegen, dem er, Bärlach, mit konventioneller Polizeiarbeit nicht beikommen konnte.

Beide Hauptfiguren der beiden Romane stehen zudem an der Schwelle von Polizeiarbeit und privater Ermittlung - Bärlach lässt sich krankheitsbedingt beurlauben, Matthäi scheidet ganz aus dem Polizeidienst aus -, und auch diese bislang in der Forschung kaum berücksichtigte Tatsache passt sich durchaus in die Geschichte der Kriminalliteratur des 20. Jahrhunderts ein. Sie wirft aber wohl auch ein erstes Licht auf die im Folgenden zu skizzierende Antwort auf die Frage nach der Poetik, die Dürrenmatt mit seinen Krimis verfolgt.

\section{Zu Dürrenmatts Poetik}

Dass beide Haupt- bzw. Ermittlerfiguren vor allem als Privatpersonen agieren, wird innerhalb der beiden Geschichten vordergründig damit erklärt, dass Bärlach todkrank ist und dass Matthäi, der eigentlich als Berater in den Nahen Osten abkommandiert werden soll, sich dem verweigert. Dahinter liegt jedoch jeweils eine existentielle Entscheidung, die sie außerhalb des Rahmens ihres Berufs verortet, sie jenseits von Gesetzen und Dienstvorschriften stellt.

Matthäi ist seinem ,Versprechen“ - gegeben ,,[b]ei meiner Seligkeit““ (Dürrenmatt 1980c, S. 32) - und seinem Gewissen verpflichtet und deshalb gegen alle Vernunft und guten Ratschläge darauf fixiert, den Mörder Gritlis zu fassen. Weil ihm das nicht gelingt, sein Plan fehlschlägt, ist auch sein Leben verfehlt.

\footnotetext{
${ }^{21}$ Hühn (1998, S. 245) beschreibt diesen Roman übrigens (ohne ihn als unzuverlässig erzählt auszuweisen) ganz im Sinne meiner Überlegungen als ,interessantes Beispiel dafür, wie zugleich von dem Erzählschema des klassischen Detektivromans extrem abgewichen und eng daran festgehalten werden kann.“ Ähnlich äußert sich später Vogt (2014, S. 72 f.).
} 
Bärlach agiert nicht nur außerhalb des Gesetzes, sondern auch gegen dieses. Sein eigener Tod steht unmittelbar bevor - die Ärzte , geben ihm noch ein Jahr ${ }^{\text {- }}$-, und so muss er, um Gastmann zu eliminieren, sein Richter werden und den Henker beauftragen. Andernfalls bliebe ihm, dem Polizisten, der andere, der Verbrecher, endgültig den einen Schritt voraus (Dürrenmatt 1980a, S. 70).

Die beiden Verbrecher der Romane könnten allerdings unterschiedlicher kaum sein: auf der einen Seite der armselige Schrott, der sich gegen seine dominante Frau nicht wehren kann und deshalb zwanghaft (Dürrenmatt 1980c, S. 159) kleine Mädchen tötet; auf der anderen Seite Gastmann, der zum freien Verbrecher geworden ist, weil er es kann, weil ihm niemand, auch Bärlach nicht, etwas nachweisen kann (Dürrenmatt 1980a, S. 68).

Bärlach ist deshalb mehr als verzweifelt nach seiner Begegnung mit Gastmann und fragt sich: „,Was ist der Mensch?““ (Dürrenmatt 1980a, S. 73). Diese anthropologische Frage ist es, die Dürrenmatts Kriminalromane - und wohl nicht nur diese - aufwerfen und diskutieren, mit allen ihren philosophischen, ethischen, juristischen, soziologischen und theologischen Teilfragen.

Dass Kriminalliteratur für die Erörterung solcher Fragen nach dem Verhältnis von Gut und Böse bzw. Verbrechen und Strafe ihrem Sujet entsprechend geeignet ist, liegt auf der Hand.

Dass ihr konventionelles Regelwerk, das allzu optimistisch ein kognitiv wie moralisch befriedigendes Ende vorsieht, kaum geeignet ist, gerade in den Jahren nach dem Zweiten Weltkrieg und dem Holocaust, sachadäquate Einschätzungen zur conditio humana zu formulieren, ist aber wohl ebenfalls nicht zu bestreiten. Dieses Dilemma zu lösen, dient deshalb der Einsatz unzuverlässigen Erzählens in den beiden Kriminalromanen Dürrenmatts: Er erlaubt es, ein detektivromankonformes Ende, in dem alle Indizien zu einer den Leser überraschenden Lösung des Kriminalfalls zusammengeführt werden, mit einer Geschichte zu verknüpfen, deren Ende nicht mit dem ,happy end“ der Auflösung des Falls einhergeht: Matthäi ist daran zerbrochen, den Fall Gritli Moser nicht gelöst zu haben. Bärlach ist, um Gastmann zu fassen, selbst zum Mordauftraggeber geworden.

„Kriminalliteratur ist nämlich eher die Literatur der Kontingenz als die Literatur irgendeines höheren Sinns. Deswegen tendiert sie, wenn sie gelungen ist, eher zu gebrochenen, ambigen, ironischen oder komischen Texten." So bestimmt Thomas Wörtche (2008, S. 14 f.) das Welt- und Menschenbild der echten, dem bloßen ,Grimmi ‘ entgegengesetzten Kriminalliteratur so ganz anders als Dürrenmatts Dr. H., aber wohl sehr im Sinne Dürrenmatts selbst.

\section{Zuverlässiges Erzählen in Der Verdacht}

Der Verdacht, Dürrenmatts zweiter Kriminalroman und vielleicht „die erste fiktive Holocaust-Erzählung eines [...] nicht jüdischen Autors“ (Battegay 2015, S. 174, Anm. 3), verfolgt ebenso offenkundig die anthropologisch aufgeladene Frage nach 
dem Wesen des Menschen und der Herkunft des Bösen in der Welt. ${ }^{22}$ Er kommt allerdings im Unterschied zu Der Richter und sein Henker und Das Versprechen ohne den Einsatz unzuverlässigen Erzählens aus, sodass abschließend zu fragen ist, wie das gelingt, ohne den Roman als Krimi zu disqualifizieren.

Bärlach liegt nach der Operation, die ihm ein weiteres Lebensjahr ermöglichen soll, im Krankenhaus. In einem Life-Magazin aus dem Jahr 1945 sieht er die Photographie eines Dr. Nehle aus dem KZ Stutthof bei Danzig und reicht es seinem Arzt, Dr. Hungertobel, mit der Bemerkung: „,Es waren Tiere““ (Dürrenmatt 1980b, S. 123). Hungertobel meint, auf dem Photo seinen ehemaligen Kommilitonen Emmenberger zu erkennen, der vermeintlich nach Chile ausgewandert, aber inzwischen zurück in die Schweiz gekommen ist und in Zürich eine Privatklinik für die High Society führt.

Der Verdacht, Nehle und Emmenberger seien ein- und dieselbe Person, erhärtet sich durch verschiedene Recherchen, die Bärlach vom Krankenbett aus führt und führen lässt, im ersten Teil des Romans. Insbesondere Bärlachs Informant Gulliver, ein Jude, der Nehle ausgesetzt gewesen ist, aber den Holocaust überlebt hat und seither konsequent unter den Menschen allein ,,zwischen Peinigern und Gepeinigten“" (Dürrenmatt 1980b, S. 158) unterscheidet, um allein Erstere zu bekämpfen, liefert dafür entscheidende Hinweise. Doch die Identitätshypothese ,Nehle ist gleich Emmenberger“ ist, so Bärlach, nur ,,das Wahrscheinliche““, deshalb aber noch nicht zwingend auch ,,das Wirkliche“" (Dürrenmatt 1980b, S. 158). Der bloß räsonierende, das Wahrscheinliche ermittelnde Detektiv Bärlach hat seine Aufgabe erledigt.

Im zweiten Teil des Romans verlässt Bärlach deshalb sein Krankenhausbett und wird zum aktiv handelnden Ermittler, der sich der Wirklichkeit, ihren Zufällen und ihren Gefahren aussetzt, um den Verbrecher zu stellen: Bärlach lässt sich inkognito in Emmenbergers Klinik einweisen. Und der Roman, der als Detektivroman begonnen hat, wird zum Thriller, in dem sich Verbrechens- und Aufklärungsgeschichte nicht mehr voneinander trennen lassen.

Die Identitätshypothese des ersten Romanteils bestätigt sich rasch. Emmenberger bietet seiner schwer reichen Klientel dieselben Dienste an wie seinen Opfern im KZ: Für die Hoffnung auf ein Weiterleben bei schwerer Krankheit lassen sich die Patienten ohne jegliche Betäubung operieren (Dürrenmatt 1980b, S. 223 f.). Es geht nun also nicht mehr darum, den Täter zu identifizieren, sondern ihn zu besiegen, ihn zu überwältigen oder auch nur ihm wieder zu entkommen. Doch genau das ist trotz aller Vorkehrungen, die Bärlach getroffen hat, nicht möglich: Er sitzt bzw. liegt in der Falle des Krankenbetts, sediert und schwer bewacht,

\footnotetext{
${ }^{22}$ Battegay (2015, S. 180) bestätigt diese Einschätzung mit spezifischem Blick auf die Shoah, wenn er schreibt, Der Verdacht könne „als theologisch-philosophische Auseinandersetzung mit den Konsequenzen der Shoah an sich gelesen werden“, zumal der „Krimi als Genre, das Moral und Erkenntnistheorie gleichermaßen beschäftigt, [...] für eine solche Auseinandersetzung prädestiniert" sei.
} 
dem Tod geweiht durch eine Emmenberger'sche Operation. Das Böse scheint gesiegt zu haben, Bärlach hat den „,Glauben an die Gerechtigkeit““ (Dürrenmatt 1980b, S. 255) bereits verloren.

In dieser Situation Bärlachs und des Romans kurz vor ihrem jeweiligen Ende könnte der Aha-Effekt einer vorherigen Lesertäuschung durch erzählerische Unzuverlässigkeit - Bärlach könnte seinen Klinikaufenthalt etwa phantasiert haben natürlich helfen, den Roman doch noch zu einem guten Ende zu führen. Doch das würde die Aussage des Romans zerstören, dass die Welt dieser Klinik die ,Wirklichkeit' ist, unsere Wirklichkeit. Dürrenmatt wählt deshalb einen anderen Weg, seinen Ermittler zu retten: den ,deus ex machina', der seit Aristoteles als ,Wunder" aus der Plotgestaltung nach den Kriterien der Wahrscheinlichkeit und Kausalität eigentlich verbannt ist: Gulliver, dessen Fortleben nach dem KZ ohnehin schon ein Wunder ist, richtet Emmenberger und rettet Bärlach gerade noch zur rechten Zeit (Dürrenmatt 1980b, S. 260).

Das Ende des Verdachts weicht also bewusst und erkennbar ab von der ,realistischen' Grundausrichtung des Kriminalromans und integriert Unwahrscheinliches, ja Phantastisches. Auch Battegay betont zu Recht, dass der Romanschluss die Figuren ,mythisch-satirisch “ überzeichnet, selbst ,symbolisch überfrachtet' ist und so ,jedes realistische Erzählen [sprengt]“. Die so erzeugte „Irrealität“ ist, so Battegay weiter, ,für den Text konstitutiv, sie dient der Satire auf eine Welt, in der immer noch so getan wird, als ob das Wirkliche auch das Vernünftige sei“" (Battegay 2015, S. 184).

Das geschieht allerdings, wie ich ergänzend zum Abschluss betonen möchte, auch zum Zweck der Erfüllung einer anderen Anforderung der Gattung Kriminalroman, nämlich der nach einem happy end. Unsere Welt bedarf, so könnte man die daraus resultierende Weltauffassung des Romans auf den Punkt bringen, der Rettung, die wir uns eigentlich nur als wundersame vorstellen können, auch wenn wir wissen, dass es Wunder nicht gibt.

\section{Literatur}

Alewyn, Richard: Anatomie des Detektivromans [1968/1971]. In: Der Kriminalroman. PoetikTheorie - Geschichte. Hg. Jochen Vogt. München: Fink / UTB, 1998, 52-72.

Arnold, Armin: Die Quellen von Dürrenmatts Kriminalromanen. In: Facetten. Studien zum 60. Geburtstag Friedrich Dürrenmatts. Hg. Gerhard P. Knapp und Gerd Labroisse. Bern u. a.: Lang, 1981, 153-174.

Aumüller, Matthias: Offenheit und Geschlossenheit als Funktionen des unzuverlässigen Erzählens. Mit Interpretationsbeispielen anhand von Texten von Ernst Weiß, Paul Zech und Stefan Zweig. In: Journal of Literary Theory 12.1 (2018), 127-150.

Battegay, Caspar: „Wahnsinn als Methode“. Friedrich Dürrenmatts Der Verdacht als Kriminalroman nach der Shoah. In: Kriminalliteratur und Wissensgeschichte. Genres - Medien Techniken. Hg. Clemens Peck und Florian Sedlmeier. Bielefeld: transcript 2015, 173-196.

Bergengruen, Maximilian: „Vergessen Sie jedoch nicht, daß die Zeit auch vor den berühmtesten Kriminalisten nicht haltmacht." Paradigmen der Kriminalistik in Friedrich Dürrenmatts Der Richter und sein Henker. In: Dramaturgien der Phantasie. Dürrenmatt intertextuell 
und intermedial. Hg. Ulrich Weber, Peter Schnyder, Peter Gasser und Peter Rusterholz. Göttingen: Wallstein, 2014, 41-60.

Broich, Ulrich: Der entfesselte Detektivroman [1978]. In: Der Kriminalroman. Poetik - Theorie - Geschichte. Hg. Jochen Vogt. München: Fink / UTB 1998, 97-110.

Christie, Agatha: The Murder of Roger Ackroyd [1926]. Dt.: Alibi. München: Goldmann, 1961.

Dürrenmatt, Friedrich: Der Richter und sein Henker [1952]. In: Friedrich Dürrenmatt: Der Richter und sein Henker / Der Verdacht. Die zwei Kriminalromane um Kommissär Bärlach. Zürich: Diogenes, 1980 (1980a), 9-117 (=Werkausgabe, Bd. 19).

Dürrenmatt, Friedrich: Der Verdacht [1953]. In: Friedrich Dürrenmatt: Der Richter und sein Henker / Der Verdacht. Die zwei Kriminalromane um Kommissär Bärlach. Zürich: Diogenes, 1980 (1980b), 119-265 (= Werkausgabe, Bd. 19).

Dürrenmatt, Friedrich: Das Versprechen. Requiem auf den Kriminalroman [1958]. In: Friedrich Dürrenmatt: Das Versprechen. Requiem auf den Kriminalroman/Aufenthalt in einer kleinen Stadt. Fragment. Zürich: Diogenes, 1980 (1980c), 9-163 (=Werkausgabe, Bd. 22).

Dürrenmatt, Friedrich: Nachwort zu ,Das Versprechen ' [1958]. In: Friedrich Dürrenmatt: Das Versprechen. Requiem auf den Kriminalroman / Aufenthalt in einer kleinen Stadt. Fragment. Zürich: Diogenes, 1980 (1980d), 203 (=Werkausgabe, Bd. 22).

Gasser, Peter: Die Geburt der Literatur aus dem Geiste des Spiels. Zu Friedrich Dürrenmatts Dramaturgie der Phantasie. In: Dramaturgien der Phantasie. Dürrenmatt intertextuell und intermedial. Hg. Ulrich Weber, Peter Schnyder, Peter Gasser und Peter Rusterholz. Göttingen: Wallstein, 2014, 19-40.

Grice, H. Paul: Logic and Conversation [1975]. Dt.: Logik und Konversation. In: Handlung, Kommunikation, Bedeutung. Hg. Georg Meggle. Frankfurt am Main: Suhrkamp 1979, 243-265.

Heyd, Theresa: Unreliability. The Pragmatic Perspective Revisited. In: Journal of Literary Theory 5.1 (2011), 3-18.

Hühn, Peter: Der Detektiv als Leser. Narrativität und Lesekonzepte im Detektivroman [1987]. In: Der Kriminalroman. Poetik - Theorie - Geschichte. Hg. Jochen Vogt. München: Fink / UTB, 1998, 239-254.

Kindt, Tom: Unzuverlässiges Erzählen und literarische Moderne. Eine Untersuchung der Romane von Ernst Weiß. Tübingen: Niemeyer 2008.

Klimek, Sonja: Unzuverlässiges Erzählen als werkübergreifende Kategorie. Personale und impersonale Erzählinstanzen im phantastischen Kriminalroman. In: Journal of Literary Theory 12.1 (2018), 29-54.

Köppe, Tilmann und Tom Kindt: Erzähltheorie. Eine Einführung. Stuttgart: Reclam 2014.

Ladenthin, Volker: Warum man heute Friedrich Dürrenmatts Roman Der Richter und sein Henker lesen sollte. In: Was wir lesen sollen. Kanon und literarische Wertung am Beginn des 21. Jahrhunderts. Hg. Stefan Neuhaus und Uta Schaffers. Würzburg: Königshausen \& Neumann 2016, 387-413.

Lang, Simone: „Zumindest hätte er sich all das gewünscht. “ Unzuverlässiges Erzählen in der Heterodiegese. Diss. Universität Bremen 2016 (im Erscheinen).

Ligensa, Annemone: Perverse suspense. Spannung und unzuverlässiges Erzählen an Beispielen angloamerikanischer Literatur. In: Zwischen Text und Leser. Studien zu Begriff, Geschichte und Funktion literarischer Spannung. Hg. Ingo Irsigler, Christoph Jürgensen und Daniela Langer. München: edition text+kritik, 2008, 50-67.

Lindemann, Uwe: Narrativik des Detektivromans. Zwei Geschehen - zwei Geschichten - ein Text. In: Orbis Litterarum 57 (2002), 31-51.

Mellmann, Katja: Empirische Emotionsforschung. In: Handbuch Literatur \& Emotionen. Hg. Martin von Koppenfels und Cornelia Zumbusch. Berlin und Boston: de Gruyter 2016, 158-175.

Neuhaus, Volker: ,... we're in a detective story, and we don't fool the reader by pretending we're not“. Das Spiel mit der Gattung als Quelle der Komik im Detektivroman. In: „Kann man denn auch nicht lachend sehr ernsthaft sein?" Sprachen und Spiele des Lachens in der Literatur. Hg. Daniel Fulda, Antje Roeben und Norbert Wichard. Berlin und New York: de Gruyter 2010, 29-44. 
Richter, Jochen: „Um ehrlich zu sein, ich habe nie viel von Kriminalromanen gehalten.“ Über die Detektivromane von Friedrich Dürrenmatt. In: Experimente mit dem Kriminalroman. Ein Erzählmodell in der deutschsprachigen Literatur des 20. Jahrhunderts. Hg. Wolfgang Düsing. Frankfurt am Main u. a.: Lang 1993, 141-153.

Riedlinger, Stefan: Tradition und Verfremdung. Friedrich Dürrenmatt und der klassische Detektivroman. 2. Aufl. Marburg: Tectum 2007.

Schmid, Wolf: Elemente der Narratologie. Berlin: de Gruyter, 2005.

Suits, Bernard: Die Detektivgeschichte: Eine Fallstudie über Spiele in der Literatur [1985]. In: Der Kriminalroman. Poetik - Theorie - Geschichte. Hg. Jochen Vogt. München: Fink / UTB, 1998, 255-273.

Thielking, Sigrid und Jochen Vogt (Hg.): ,Beinahekrimis ‘ - Beinahe Krimis!? Bielefeld: Aisthesis, 2014.

Tschimmel, Ira: Kritik am Kriminalroman. In: Facetten. Studien zum 60. Geburtstag Friedrich Dürrenmatts. Hg. Gerhard P. Knapp und Gerd Labroisse. Bern u. a.: Lang, 1981, 175-190.

Van Dine, S. S. [Willard Huntington Wright]: Twenty Rules for Writing Detective Stories [1928]. Dt.: Zwanzig Regeln für das Schreiben von Detektivgeschichten. In: Der Kriminalroman I. Zur Theorie und Geschichte einer Gattung. Hg. Jochen Vogt. München: Fink / UTB, 1971, 143-147.

Vogt, Jochen: Krimis, Antikrimis, „Gedanken“-Krimis. Wie Friedrich Dürrenmatt sich in ein gering geschätztes Genre einschrieb. In: ,Beinahekrimis“ - Beinahe Krimis!? Hg. Sigrid Thielking und Jochen Vogt. Bielefeld: Aisthesis, 2014, 51-73.

Waldmann, Günter: Kriminalroman - Anti-Kriminalroman. Dürrenmatts Requiem auf den Kriminalroman und die Anti-Aufklärung. In: Der Kriminalroman I. Zur Theorie und Geschichte einer Gattung. Hg. Jochen Vogt. München: Fink/UTB, 1971, S. 206-227.

Wigbers, Melanie: Krimi-Orte im Wandel. Gestaltung und Funktionen der Handlungsschauplätze in Kriminalerzählungen von der Romantik bis in die Gegenwart. Würzburg: Königshausen \& Neumann 2006.

Wörtche, Thomas: Kriminalliteratur tanzt, schwimmt und rudert auf vielerlei Grenzlinien. Ein Vorwort. In: Thomas Wörtche: Das Mörderische neben dem Leben. Ein Wegbegleiter durch die Welt der Kriminalliteratur. Lengwil: Libelle 2008, 7-19.

Es geschah am hellichten Tag. Reg. Ladislao Vajda. Praesens-Film, CCC-Film, Chamartín-SA, 1958.

The Pledge (dt.: Das Versprechen). Reg. Sean Penn. Warner Bros., 2001.

Open Access Dieses Kapitel wird unter der Creative Commons Namensnennung 4.0 International Lizenz (http://creativecommons.org/licenses/by/4.0/deed.de) veröffentlicht, welche die Nutzung, Vervielfältigung, Bearbeitung, Verbreitung und Wiedergabe in jeglichem Medium und Format erlaubt, sofern Sie den/die ursprünglichen Autor(en) und die Quelle ordnungsgemäß nennen, einen Link zur Creative Commons Lizenz beifügen und angeben, ob Änderungen vorgenommen wurden.

Die in diesem Kapitel enthaltenen Bilder und sonstiges Drittmaterial unterliegen ebenfalls der genannten Creative Commons Lizenz, sofern sich aus der Abbildungslegende nichts anderes ergibt. Sofern das betreffende Material nicht unter der genannten Creative Commons Lizenz steht und die betreffende Handlung nicht nach gesetzlichen Vorschriften erlaubt ist, ist für die oben aufgeführten Weiterverwendungen des Materials die Einwilligung des jeweiligen Rechteinhabers einzuholen. 